\title{
Procesos de desarrollo del talento humano en una clínica de especialidades de Pasto, Colombia
}

\author{
Human talent development processes in a specialty clinic in Pasto, Colombia
}

Jeraldine Milena Matabajoy-Montilla1* orcid.org/0000-0003-0840-6534

Sonia Maritza Matabachoy-Tulcán 1,2 orcid.org/0000-0002-3681-8440

Lina Maria Obando-Guerrero1,3 orcid.org/0000-0001-6286-5251

1 Programa de Psicología, Universidad de Nariño. Pasto, Colombia

2 Grupo de investigación CONEPSI Programa de Psicología, Universidad de Nariño. Pasto, Colombia

3 Grupo de investigación Libres Pensadores, Universidad de Nariño. Pasto, Colombia

Fecha de recepción: Agosto 23 - $2017 \quad$ Fecha de revisión: Septiembre 4 - $2017 \quad$ Fecha de aceptación: Diciembre 21 - 2017

Matabajoy-Montilla JM. Matabachoy-Tulcán SM, Obando-Guerrero LM. Procesos de desarrollo del talento humano en una clínica de especialidades de Pasto- Colombia. Univ. Salud. 2018;20(1):26-36. DOI: http://dx.doi.org/10.22267/rus.182001.106

\section{Resumen}

Introducción: Los trabajadores en la organización desempeñan un papel fundamental en el cumplimiento de los objetivos corporativos, en ese sentido, es importante identificar los procesos de desarrollo de talento humano en el sector salud puesto que éstos contribuyen en el desarrollo humano y organizacional. Objetivo: Se caracterizó los procesos de desarrollo de talento humano capacitación, calidad de vida laboral, salud laboral, clima organizacional y evaluación de desempeño en una clínica de especialidades de la ciudad de Pasto- Colombia. Materiales y métodos: El estudio se abordó desde el paradigma cuantitativo de tipo descriptivo con una muestra de 211 colaboradores. Se construyó la escala denominada "Percepción de los procesos de desarrollo del talento humano", cuyo alfa de Cronbach fue de 0,97. Resultados: Se encontraron diferencias estadísticamente significativas en la categoría de capacitación (en las subcategorías detección de las necesidades, plan y programas, evaluación de la capacitación, seguimiento), calidad de vida laboral (en las subcategorías significado del trabajo, compromiso laboral) y evaluación de desempeño (en las subcategorías métodos y resultados). Conclusiones: Se reconoce la necesidad de continuar fortaleciendo los procesos de capacitación, calidad de vida laboral y evaluación de desempeño.

Palabras clave: Capacitación; salud laboral; rendimiento laboral; satisfacción en el trabajo; análisis del desempeño. (Fuente: DeCS, Bireme).

\section{Abstract}

Introduction: Workers in the organization play a fundamental role in meeting the corporate objectives. In this sense, it is important to identify the processes of human talent development in the health sector since they contribute to human and organizational development. Objective: The processes of human talent development training, quality of work life, occupational health, organizational climate and performance evaluation were characterized in a specialty clinic in the city of Pasto, Colombia. Materials and methods: The study was addressed from the quantitative paradigm of descriptive type with a sample of 211 collaborators. The scale called "perception of human talent development processes" was built, whose Alfa de Cronbach was 0.97. Results: The results were systematized in the statistical program IBM SPSS statistics. Statistically significant differences were found in the training category (in the categories of needs detection, plan and programmes, training evaluation and follow-up), quality of working life (in the subcategories: meaning of work and labor commitment) and performance evaluation (in the subcategories: methods and results). Conclusions: The need to continue strengthening the processes of training, quality of work life and evaluation of performance is recognized.

\footnotetext{
*Autor de correspondencia Jeraldine Milena Matabajoy Montilla 
Keywords: Education; occupational health; work performance; job satisfaction; employee performance appraisal. (Source: DeCS, Bireme).

\section{Introducción}

Las organizaciones procuran mejorar las capacidades, competencias y habilidades de su talento humano, puesto que del aporte que los trabajadores hacen a la organización depende la efectividad de sus procesos. Es así como la clave de las áreas del talento es brindar elementos para desarrollo profesional que estén alineados a la estrategia organizacional para dar cumplimiento al logro de los objetivos corporativos(1). En ese sentido una de las funciones misionales del área es desarrollar procesos articulados que generen desarrollo humano y organizacional, que se logra a través de los procesos de administración (selección, contratación, inducción) y desarrollo del talento humano (capacitación, calidad de vida laboral, salud laboral, clima organizacional y evaluación de desempeño).

En el presente estudio se abarcaron las categorías de los procesos de desarrollo de talento humano como son la capacitación; que incluyó las subcategorías de detección, planes y programas de capacitación, evaluación y seguimiento; calidad de vida laboral constituida por las subcategorías familia, satisfacción laboral, significado del trabajo y compromiso laboral; salud laboral conformada por factor psicosocial, estilo de vida y tiempo libre; clima organizacional formada por las subcategorías de estilos de dirección, relaciones interpersonales, participación y finalmente evaluación de desempeño que aborda resultados y métodos.

La capacitación es un proceso continuo de enseñanza-aprendizaje mediante el cual se desarrolla las habilidades y destrezas de los trabajadores, lo que les permite un mejor desempeño en sus labores habituales. En la organización se acude al proceso de capacitación para que los colaboradores desarrollen nuevas habilidades y destrezas que podrán poner en práctica en su campo laboral; de ahí la importancia de evaluar cuáles son las principales demandas de capacitación al interior de las organizaciones $^{(2)}$.

Las subcategorías que corresponden a esta categoría son la detección de necesidades relacionada con la posibilidad de generar un diagnóstico claro sobre las áreas y temas a capacitar; los planes y programas de capacitación enfocados en procesos estructurados a ejecutar en la capacitación; la evaluación y seguimiento de la capacitación que permite conocer la efectividad y la aplicabilidad del conocimiento adquirido al logro de los objetivos. Estos elementos pueden ser considerados en la elaboración de un plan estructurado para la capacitación continua en clínicas y hospitales(3).

La calidad de vida laboral (CVL), hace referencia al grado de satisfacción y bienestar físico, psicológico y social experimentado por las personas en su puesto de trabajo, por ello, abarca una doble dimensión: objetiva y subjetiva. La primera se relaciona con las condiciones económicas, jurídicas, sociales, ecológicas, materiales y técnicas de trabajo y la segunda está asociada a las percepciones y valoraciones relativas a la propia experiencia laboral y a su contribución al desarrollo personal( ${ }^{(4)}$. Dentro de las subcategorías que integran la calidad de vida laboral se encuentra en primer lugar la familia la cual es considerada como el sistema social primario del individuo y a nivel organizacional es un eje central que puede enriquecer o alterar la satisfacción y el compromiso laboral(5).

La satisfacción laboral puede definirse como una respuesta emocional positiva hacia el puesto de trabajo(6). El significado del trabajo corresponde al conjunto de valores y creencias relacionadas con él. De acuerdo con Blanch(4), esos significados y valores varían según los factores ambientales y personales o condiciones como el salario, tipo de contrato, suficiencia de recursos materiales, técnicos e instrumentales, los riesgos para la salud, la integridad física, entre otros. El compromiso laboral según Yepes et al.(7), 
corresponde al vínculo psicológico y emocional que el colaborador tiene hacia su lugar de trabajo, que influye en el deseo de permanecer en la organización teniendo un sentido de lealtad y deber.

La categoría clima organizacional se ha definido como el conjunto de percepciones que el trabajador construye sobre su ambiente laboral en relación a las políticas, prácticas y procedimientos organizacionales, ya sean formales o informales, las cuales determinan los comportamientos de los individuos y su desempeño en la organización( ${ }^{(8)}$. Dentro de las subcategorías que conforman el clima organizacional se encuentra los estilos de dirección que se refiere a la forma y estilo de liderazgo propias de cada empresa y los modos de gestión de los trabajadores. La elección de un estilo de liderazgo en particular podría ser un factor de gran relevancia en el aumento de la satisfacción laboral de los colaboradores, de manera que cada uno de ellos pueda lograr el máximo desarrollo posible de sus habilidades intrínsecas, alcanzando así, una alta eficiencia para ellos mismos y para su equipo(9).

Las relaciones interpersonales se dan entre dos o más compañeros y áreas de trabajo, pueden ser relaciones de apoyo y reciprocidad, o bien relaciones conflictivas que afectan la satisfacción laboral, el desempeño individual, el bienestar general de los trabajadores y en general el clima organizacional, por lo tanto, se resalta el papel del trabajador y su relación con el otro en el ámbito laboral(10).

La subcategoría de participación es la percepción que tiene el trabajador sobre la apertura de la empresa con respecto a los aportes que puedan hacerse sobre el trabajo que desempeña, la dinámica laboral o cualquier otro proceso decisorio en el que el colaborador pueda contribuir ${ }^{(9)}$; este factor se produce en el marco de una cultura organizacional flexible, donde el liderazgo es abierto y se brinda la oportunidad de participar en la toma de decisiones(11).

En relación a la categoría salud laboral es importante mencionar que esta se construye en un ambiente de trabajo adecuado, con condiciones de trabajo justas, donde los trabajadores puedan desarrollar una actividad con dignidad y donde sea posible su participación para la mejora de las condiciones de salud y seguridad. Esta categoría está integrada por las subcategoría factores psicosociales, los cuales son las interacciones entre las condiciones de trabajo, las capacidades, necesidades y expectativas del trabajador que están influenciadas por las costumbres, la cultura y por las condiciones personales fuera del trabajo(12).

Los estilos de vida son conductas que se asumen frente a situaciones y son susceptibles de modificación; estos se relacionan con alimentación, actividad física, uso de elementos de protección y actividades recreativas, entre otras. El tiempo libre también llamado ocio o tiempo fuera del trabajo es una dimensión importante en la calidad de vida laboral, puesto que favorece la salud mental y contribuye no sólo a prevenir la enfermedad, sino también a disminuir los costos y obtener beneficios físicos y económicos(13).

Finalmente, se encuentra la categoría de evaluación de desempeño la cual es un proceso administrativo de gestión individual y colectiva que pretende identificar, evaluar y calificar el desempeño de funciones $y$ compromisos laborales y comportamentales que permitan el logro de las metas corporativas(14). Según Sánchez y Calderón(15) la evaluación de desempeño se desarrolla mediante etapas de aplicación permanente y sistemática, dado que, a través de ésta, la organización puede adaptarse a los cambios que se producen en el entorno y afectan de manera directa sus dinámicas y a sus trabajadores, por tanto, es de gran importancia la evaluación permanente de las funciones laborales. Además, es necesario no sólo centrarse en sus aspectos de mejora sino además en sus fortalezas, de igual forma la evaluación posibilita detección de necesidades de capacitación, reconocimiento de competencias, retroalimentación del desempeño y revisión de la adecuación de personas a los cargos. 
Esta categoría está formada por la subcategoría de métodos y resultados, la cual se relaciona con todos los procesos, estrategias y formas para llevar a cabo el proceso; existen diferentes métodos de supervisión (observación, recorrido de supervisión, entrevistas y enseñanzas), que tienen como meta principal valorar las habilidades intelectuales y técnicas, en la realización de las funciones diarias por el personal colaborador. La sub categoría comprende toda la información obtenida y el uso que se le da a la misma para la construcción de planes de mejora(1).

A partir de la revisión expuesta surge el interés de caracterizar los procesos de desarrollo de talento humano en una clínica de especialidades de alta y mediana complejidad de la ciudad de Pasto dado que el área de talento humano se encuentra en proceso de fortalecimiento como parte de su plan estratégico.

\section{Materiales y métodos}

El estudio se desarrolló desde el paradigma de investigación cuantitativo, de tipo descriptivo. La muestra estuvo constituida por 211 colaboradores de un total de 270, quienes se seleccionaron mediante un muestreo aleatorio simple.

El instrumento que se construyó fue una escala tipo Likert denominada "Percepción de los procesos de desarrollo de talento humano". El análisis técnico de la escala incluyó el análisis de validez y confiabilidad. Para el proceso de validez se obtuvo evidencias de contenido mediante el apoyo de 3 jueces expertos en el campo de la psicología organizacional, de la medición y evaluación psicológica. Para la obtención de validez de constructo la escala fue sometida al análisis factorial exploratorio, encontrando 5 factores: capacitación, calidad de vida laboral, clima organizacional, salud laboral y evaluación de desempeño que explican el 55,6\% de la varianza total.

Para el análisis de la confiabilidad se utilizó el programa IBM SPSS Statistics obteniendo un Alfa de Cronbach de 0,97. La escala quedo conformada por 135 ítems con cinco opciones de respuesta que van de 0 (Nunca) a 4 (Siempre). Para establecer los baremos se calcularon percentiles con puntos de corte que se clasifican en bajo (0343 ), medio (344-395) y alto (396-540).

\section{Consideraciones éticas}

La investigación respetó los parámetros éticos para estudios con seres humanos contenidos en la Resolución 008430 de 1993 del Ministerio de Salud de Colombia, la Declaración de Helsinki y la Ley 1090 de 2006 en la cual se instituye el Código Deontológico y Bioético del profesional de la psicología en Colombia, que establece que el manejo de información es estrictamente confidencial, por lo tanto, el análisis y publicación de este artículo se realiza de forma general sin evidenciar información personal, con el fin de proteger la identidad de los participantes. Se contó además con la autorización de los trabajadores de la clínica a través de la firma del consentimiento informado y con el aval de la empresa donde se recolectó la información y la supervisión del área de talento humano.

\section{Resultados}

Los datos sociodemográficos indican que los participantes fueron en un $67,2 \%$ hombres y en $32,8 \%$ mujeres, cuyas edades oscilaron entre los 19 y 60 años de edad. El 44,3\% de los colaboradores son solteros, seguidos por personas casadas $(34,3 \%)$ y en un porcentaje más bajo las personas divorciadas (6\%). De igual forma se evaluó el ámbito familiar donde se identificó que los participantes con hijos representan el 70,6\%, de los cuales el 41,3\% tienen un solo hijo y en menor proporción tienen de 3 a 4 hijos (5\%). Se identificó que el 95,5\% de los trabajadores viven en el sector urbano, en viviendas familiares (33,8\%), arrendadas $(30,8 \%)$ y en anticresis (5\%), ubicándose diferencialmente en los estratos 1 (7,5\%), 2 (26,9\%), $3(32,3 \%), 4(11,4 \%)$ y $5(1,5 \%)$.

En cuanto al nivel de escolaridad, los participantes tienen un nivel técnico $(34,8 \%)$ seguido por el nivel profesional $(33,8 \%)$, tecnológico $(15,4 \%)$ y nivel de maestría $(1 \%)$. Asimismo, se encontró que el $66,2 \%$ de la muestra 
ha laborado en cargos asistenciales mientras que el $30,2 \%$ lo ha hecho en cargos administrativos. Respecto al tiempo de vinculación en la organización, el $55,7 \%$ de los colaboradores ha trabajado entre 0 a 2 años y un $35,3 \%$ durante 3 a 5 años.
Para el análisis de resultados, se calcularon estadísticos descriptivos para cada subcategoría del instrumento de medición denominado "Percepción de los procesos de desarrollo de talento humano", a partir del cual se obtuvo los resultados que se muestran en la Tabla 1.

Tabla 1. Estadísticos descriptivos para cada subcategoría de la escala "Percepción de los procesos de desarrollo de talento humano"

\begin{tabular}{|c|c|c|c|c|c|c|}
\hline & $\mathbf{N}$ & Media & $\begin{array}{l}\text { Desviación } \\
\text { estándar }\end{array}$ & Mediana & Mínimo & Máximo \\
\hline $\begin{array}{l}\text { Detección de las } \\
\text { necesidades }\end{array}$ & 201 & 9,44 & 3,524 & 10 & 0 & 16 \\
\hline Plan y programas & 201 & 6,03 & 7,066 & 6 & 0 & 102 \\
\hline $\begin{array}{l}\text { Evaluación de la } \\
\text { capacitación }\end{array}$ & 201 & 17,38 & 14,688 & 18 & 1 & 210 \\
\hline Seguimiento & 201 & 5,03 & 2,169 & 5 & 0 & 8 \\
\hline Familia & 201 & 29,16 & 48,266 & 18 & 6 & 303 \\
\hline Satisfacción laboral & 201 & 18,74 & 5,261 & 20 & 0 & 24 \\
\hline Significado del trabajo & 201 & 20,00 & 5,436 & 22 & 0 & 24 \\
\hline Compromiso laboral & 201 & 17,69 & 3,884 & 19 & 3 & 23 \\
\hline Factor psicosocial & 201 & 47,87 & 9,906 & 48 & 7 & 69 \\
\hline Estilos de vida & 201 & 23,83 & 5,922 & 25 & 0 & 32 \\
\hline Tiempo libre & 201 & 15,04 & 3,770 & 15 & 2 & 24 \\
\hline Estilos de dirección & 201 & 55,54 & 16,634 & 56 & 6 & 86 \\
\hline Relaciones interpersonales & 201 & 34,88 & 8,596 & 36 & 0 & 52 \\
\hline Participación & 201 & 12,24 & 4,289 & 12 & 0 & 20 \\
\hline Métodos & 201 & 6,26 & 3,726 & 6 & 0 & 12 \\
\hline Resultados & 201 & 11,46 & 3,637 & 12 & 0 & 16 \\
\hline
\end{tabular}

Para realizar el análisis de datos teniendo en cuenta las variables género, cargo y nivel de escolaridad de los colaboradores de la Clínica, en primer lugar, se aplicó la prueba de normalidad de Kolmogorov Smirnov, la cual arrojó un valor $\mathrm{p}$ de 0,001 , que indica que los resultados de la escala no se ajustaron a una distribución normal, por tal razón se procedió a realizar el análisis de comparación de grupos, utilizando pruebas no paramétricas.
Frente a la variable género no se encontraron diferencias estadísticamente significativas en ninguna de las subcategorías del instrumento, ya que los valores $\mathrm{p}$ obtenidos en la prueba $\mathrm{W}$ de Mann - Whitney fue mayor a 0,05 para cada una de ellas. Sin embargo, en la figura 1 se puede observar que en algunas subcategorías como "familia" los hombres presentan una media más alta $(31,70 \dot{X})$ que las mujeres $(23,95 \dot{X})$, mientras que en "evaluación de la capacitación" es el género femenino quien presenta una media más alta $(19,67 \dot{X})$ que el masculino $(16,27 \dot{X})$. 


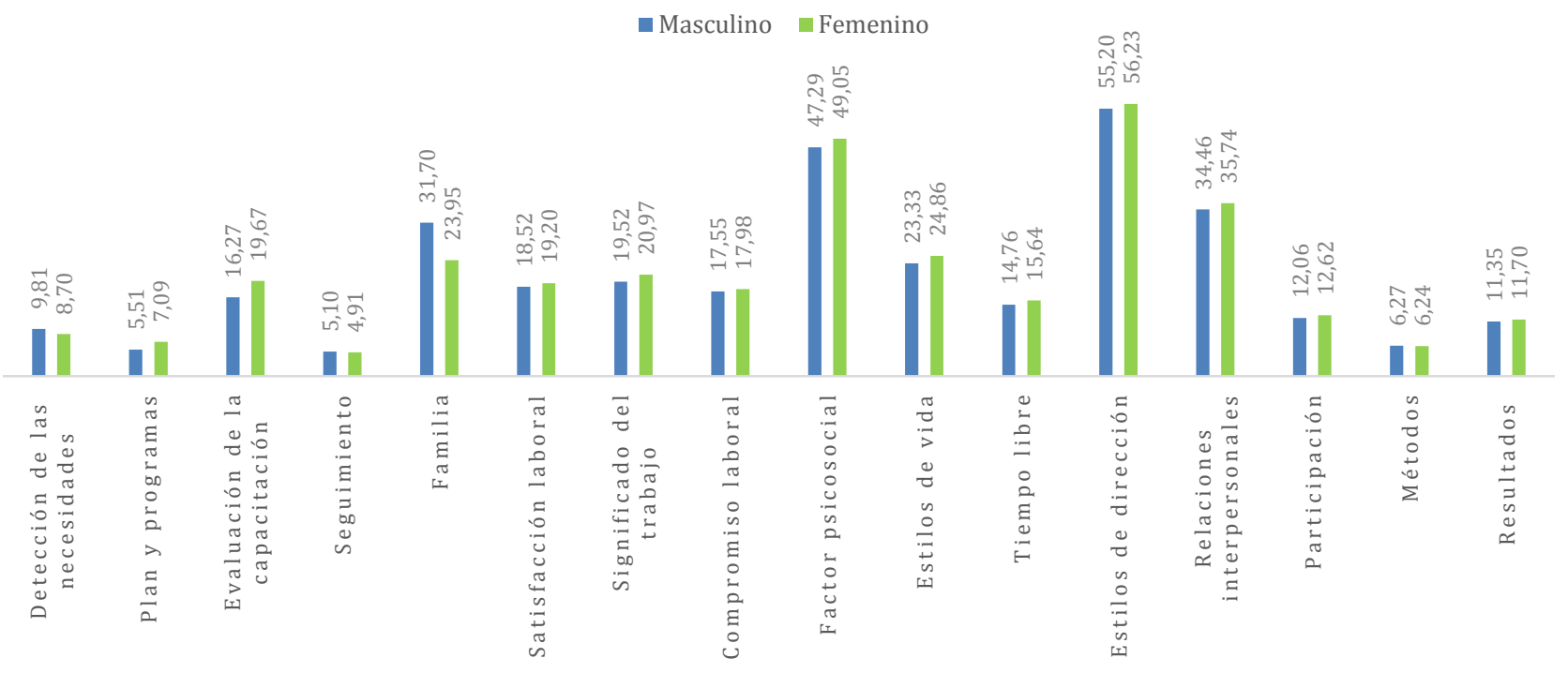

Figura 1. Media de las subcategorías de acuerdo a la variable género

En cuanto al cargo ocupado por los participantes de la investigación, la prueba W de Mann Whitney de comparación de medias mostró diferencias estadísticamente significativas en las subcategorías detección de las necesidades, plan y programas, evaluación de la capacitación y seguimiento correspondientes a la categoría Capacitación, significado del trabajo y compromiso laboral correspondientes a la categoría Calidad de vida laboral, métodos y resultados correspondientes a la categoría Evaluación del desempeño. (Tabla 2).

Tabla 2. Subcategorías que presentaron diferencias estadísticamente significativas frente a la variable cargo

\begin{tabular}{rrrrrrrr}
\hline $\begin{array}{c}\text { Detección de las } \\
\text { necesidades }\end{array}$ & $\begin{array}{c}\text { Plan y } \\
\text { programas }\end{array}$ & $\begin{array}{c}\text { Evaluación de la } \\
\text { capacitación }\end{array}$ & Seguimiento & $\begin{array}{c}\text { Significado del } \\
\text { trabajo }\end{array}$ & $\begin{array}{c}\text { Compromiso } \\
\text { laboral }\end{array}$ & Métodos & Resultados \\
\hline 2628,500 & 2813,500 & 2625,000 & 2787,500 & 3271,000 & 3295,500 & 2830,500 & 2867,500 \\
$-3,949$ & $-3,484$ & $-3,954$ & $-3,530$ & $-2,187$ & $-2,124$ & $-3,389$ & $-3,297$ \\
, 000 &, 000 &, 000 &, 000 &, 029 &, 034 &, 001 &, 001 \\
\hline
\end{tabular}

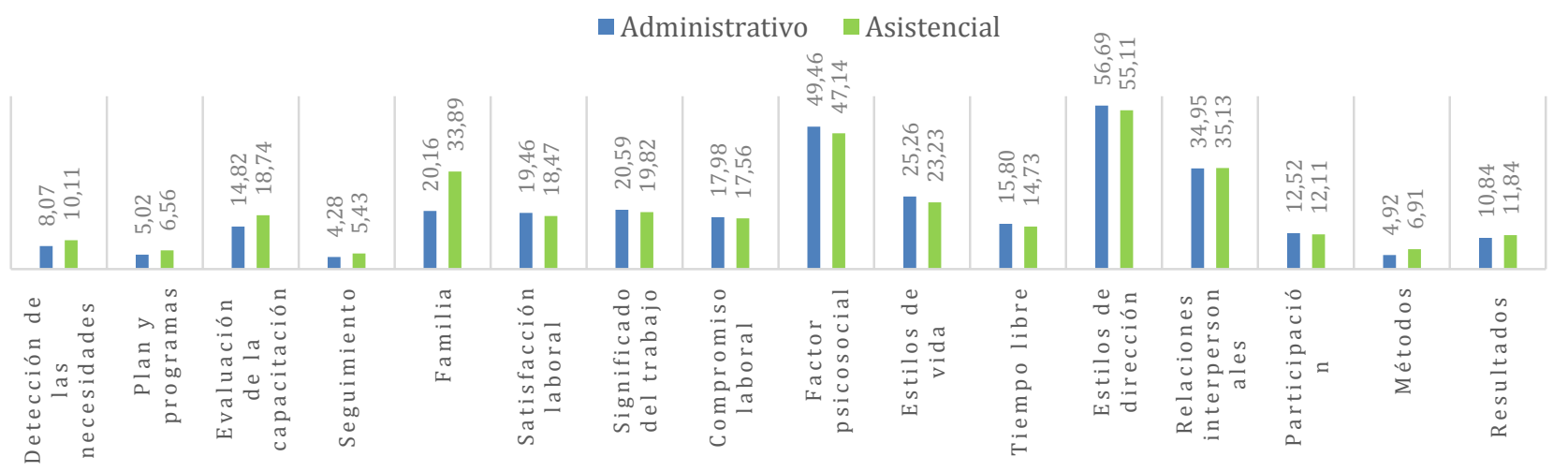

Figura 2. Media de las subcategorías distribuido según el cargo del personal de la clínica 
En la Figura 2 se muestran las medias obtenidas en cada una de las subcategorías de la prueba por los participantes del nivel asistencial y administrativo, en la cual se observan diferencias en subcategorías como evaluación de la capacitación, donde se observa una media más elevada en el nivel asistencial $(18,74 \dot{X})$ que en el administrativo $(14,82 \dot{X})$, mientras que en "factor psicosocial" se observa que el nivel administrativo presenta una media más alta $(49,46 \dot{X})$ que el nivel asistencial $(47,14 \dot{X})$.

También se calcularon las diferencias estadísticamente significativas de acuerdo al nivel de escolaridad de los participantes del estudio, lo cual se logró por medio de la prueba de Kruskall Wallis, encontrando diferencias en las subcategorías de plan y programas $(7,641)$ con un p valor de 0,022 y estilos de dirección $(6,260)$ con un $p$ valor de 0,044 .

En la Figura 3 se pueden observar las medias obtenidas en cada subcategoría, teniendo en cuenta la variable escolaridad de la muestra; en dicha figura se evidencian las diferencias en las subcategorías presentadas en la anterior tabla, en la subcategoría "Plan y programas" se presenta una media más elevada en el nivel de secundaria $(15,30 \dot{X})$ que en los niveles técnico o tecnológico $(5,81 \dot{X})$ y el nivel de estudios superiores $(5,24 \dot{X})$. Por otra parte, en la subcategoría "Estilos de dirección" se observa una media más alta en los participantes con estudios superiores $(58,49 \dot{X})$ que en los participantes con educación técnica o tecnológica $(53,45 \dot{X})$ y secundaria $(50,10 \dot{X})$.

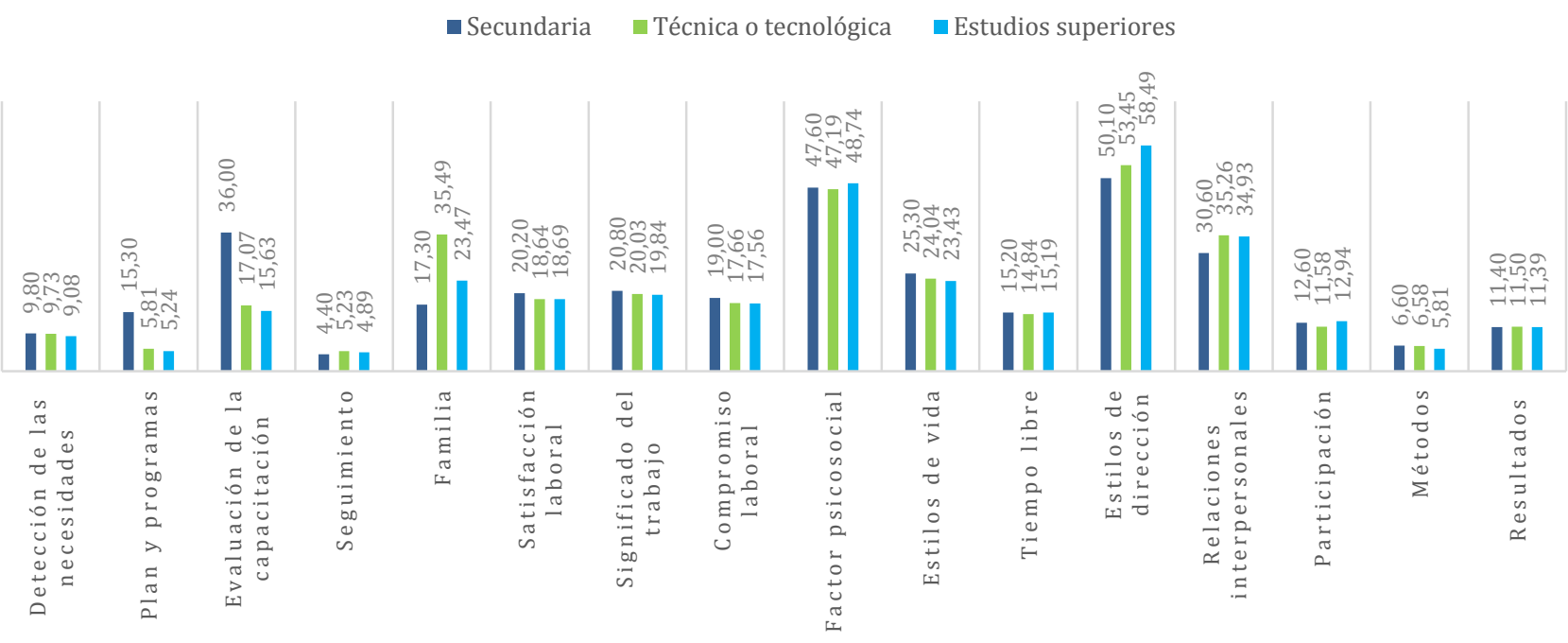

Figura 3. Media de las subcategorías distribuido según la escolaridad del personal de la Clínica

\section{Discusión}

El proceso de capacitación conformado por las subcategorías detección de las necesidades $(10,1$ $\dot{X})$, plan y programas $(6,6 \dot{X})$, evaluación de la capacitación $(18,7 \dot{X})$ y seguimiento $(5,4 \quad \dot{X})$ obtiene una media más alta en el personal asistencial, por lo que se identifica que los colaboradores reconocen la importancia de la capacitación en su desarrollo profesional. Desde esta postura Araneda, et al.(16), refieren que el mayor valor agregado de una organización está conformado por su personal, por tanto, un componente humano capacitado puede llevar a cabo las tareas de mejor forma, es decir, una empresa existe en relación con la formación del personal que la conforma.

Se identifica que el personal asistencial de la clínica reconoce y participa en la detección de necesidades en su lugar de trabajo y considera que son suficientes las estrategias que utiliza la clínica para identificar las necesidades de capacitación, presentando así una media de 10,1 
$\dot{X}$; información que se relaciona con un estudio realizado por Franco et al. (3), quienes consideran que en las instituciones prestadoras de servicios en salud, es necesario un continuo proceso de detección de necesidades para capacitar a los colaboradores, dado que se presenta continuamente rotación del personal, se adquieren nuevas tecnologías y se requiere el conocimiento en el manejo de equipos que se emplean en contextos hospitalarios.

Posterior a esta fase se encuentra la segunda subcategoría denominada plan y programas, la cual obtuvo una media de $6,6 \dot{X}$ por parte del personal asistencial de la clínica, quienes reconocen que las capacitaciones que se realizan hacen parte de un proceso articulado a los objetivos de la clínica. Teniendo en cuenta que la planificación general de la capacitación en una organización implica, seleccionar las acciones más apropiadas para atender cada necesidad, evaluar el conjunto de las propuestas de capacitación, seleccionar aquellas que serán incluidas en el plan general y finalmente elaborar el plan y el presupuesto general de capacitación, de tal manera que se contribuya al cumplimiento de objetivos corporativos tal como lo afirma Castañeda, et al.(17), quienes proponen que para lograrlo se requiere de un diseño, una ejecución y medición de resultados que sean coherentes con la estrategia empresarial, así como de la identificación adecuada de las necesidades de capacitación en función de los requerimientos del puesto, y de la creación de condiciones propicias para que, una vez concluida la acción de capacitación, el trabajador tenga las condiciones para la transferencia de lo aprendido en su labor.

Como tercera y cuarta subcategorías se encuentran la evaluación (media de 18,7 Ẋ) y seguimiento de la capacitación (media de 5,4) X் por parte del personal asistencial, quienes perciben que el conocimiento y desempeño adquirido en las capacitaciones brindadas por la clínica son evaluados y reconocen que realizan la respectiva autoevaluación del proceso. Acerca del de la capacitación, perciben que sus jefes y el personal del área de talento humano realizan el seguimiento a este proceso. Frente a esto se refiere que, por medio de las capacitaciones se adquieren conocimientos, habilidades y destrezas que son necesarias para lograr la eficacia y la excelencia en la realización de tareas, funciones y responsabilidades.

En específico, la capacitación es un proceso educacional de carácter estratégico, aplicado de manera organizada y sistemática iniciando con una detección de necesidades y finalizando con un seguimiento, lo cual facilita medir el impacto por medio de procesos sistemáticos y rigurosos que permiten evidenciar si se presentan cambios en el personal a partir de la capacitación para así mejorar el nivel de vida de los individuos y optimizar la calidad de una organización, generando de este modo, beneficios mutuos(18).

En cuanto a la evaluación de la CVL, Blanch(4) plantea que se debe tener en cuenta las dimensiones objetivas y subjetivas; en su estudio encontró que en los hospitales iberoamericanos existe una valoración positiva en cuanto a las condiciones materiales, técnicas y sociales de trabajo, así como del grado de bienestar laboral, con lo cual se identifica la importancia de la percepción de los trabajadores, lo que contribuye de manera directa a su desarrollo personal y a un aporte positivo para el crecimiento de la organización.

Entre las subcategorías de la CVL que presentaron diferencias estadísticamente significativas, se encuentra en primera instancia el compromiso laboral, el cual presentó una media de 18 X por parte del personal administrativo, quienes perciben que al interior de la clínica existe un compromiso con las metas de la organización. Asimismo, existe un sentido de pertenencia y motivación por realizar las funciones laborales, reconociendo que la organización aporta al crecimiento del colaborador. Frente a esta subcategoría Yepes et al.(7), plantean que el compromiso laboral depende del vínculo que exista entre los colaboradores y la clínica, el cual será más fuerte si los sujetos sienten que están dispuestos a esforzarse en su actividad laboral (motivación), que su desempeño no afecta su bienestar, que desarrollan un vínculo psicológico con su organización (compromiso), que se sienten miembros de esta, están orgullosos por 
pertenecer a ella y deseen seguir siendo miembros (identificación). Todas estas variables influyen en el bienestar del sujeto y en el mejoramiento de la calidad de vida laboral.

En relación a la segunda subcategoría significado del trabajo, se encontró que los participantes consideran que la clínica ofrece posibilidades de desarrollo y progreso cuando se involucran con los procesos de la organización, determinando que las funciones que desempeñan y el cargo que ocupan aportan al cumplimiento de los objetivos organizacionales. En esta subcategoría se obtuvo una media de 20,6 $\dot{X}$ por parte del personal administrativo quien presenta una media más alta en comparación con el asistencial. De acuerdo a lo propuesto por Quintana et al.(19), el significado y satisfacción laboral de los trabajadores dependerá de las condiciones ambientales en las que se encuentren expuestos por tanto es necesario garantizarles una seguridad laboral, oportunidades de desarrollo personal y una sensación subjetiva de bienestar dado que esto influye directamente en la calidad de vida laboral de los sujetos.

Respecto a la evaluación de desempeño es considerada hoy en día como una herramienta esencial para quienes tienen la responsabilidad de gerenciar el talento humano en las organizaciones. En consecuencia, para poder lograr un buen desarrollo empresarial y de las funciones establecidas en las organizaciones, es indispensable contar con un talento humano idóneo y para ello, desarrollar mecanismos capaces de reconocer, acompañar y orientar al recurso humano en su desarrollo laboral(13).

Entre las subcategorías que presentaron diferencias estadísticamente significativas se encuentra en primer lugar los métodos, la cual presentó una media de 6,9 Xं siendo más alta en el personal asistencial que en el administrativo. En un estudio realizado por López, et al.(1), se identificó la importancia del método de medición en la evaluación de desempeño, dado que de acuerdo a las características y funciones de los colaboradores se pueden asignar unos resultados y emplear planes de mejoramiento. Desde esta perspectiva, los participantes del estudio refirieron que la forma de medir su desempeño es mediante evaluaciones de sus capacidades actuales, la adecuación de su lugar de trabajo y su desempeño en el mismo, el cumplimiento de las responsabilidades y el esfuerzo empleado.

Finalmente, en la subcategoría resultados se encontró que los participantes tienen claro qué es lo que se busca obtener con el trabajo que ejecutan y la influencia de la clínica en mejorar el desempeño laboral, por tanto, la media fue de $11,8 \dot{X}$ en el personal asistencial. En ese sentido se considera que para lograr el crecimiento de la organización es de gran importancia generar cultura de evaluación permanente de las funciones que cumple el trabajador.

\section{Conclusiones y recomendaciones}

Por medio de la presente investigación se logró caracterizar los procesos de desarrollo de talento humano, de igual forma se reconoció la necesidad de continuar fortaleciendo los procesos de capacitación, calidad de vida laboral y evaluación de desempeño.

Se logró evidenciar la percepción que tienen los colaboradores de la clínica sobre el proceso de capacitación, identificando la importancia que le dan a la detección de necesidades, la creación de planes y programas de capacitación, la evaluación pertinente de dichos procesos y el seguimiento de las capacitaciones, pues es necesario verificar si los conocimientos que se imparten en las capacitaciones son aplicados en la dinámica del trabajo.

En la medición de la CVL, se identificó la relevancia que dan los colaboradores a las subcategorías significado del trabajo y compromiso laboral, por lo cual es necesario continuar fortaleciendo estos procesos para contribuir al bienestar y a la percepción que tienen de su lugar de trabajo, dado que puede influir en su rendimiento laboral y esto a su vez se verá reflejado en la productividad y calidad del servicio ofrecido a los usuarios.

Para fortalecer los procesos de talento humano se sugiere que se continúen realizando actividades 
académico-investigativas desde la psicología y otras disciplinas afines, donde se trabaje conjuntamente en la identificación, creación de estrategias para continuar generando, manteniendo y promoviendo procesos de desarrollo de talento humano, los cuales benefician a los colaboradores y la organización.

Se recomienda generar procesos de investigación de tipo correlacional que permitan ampliar la perspectiva de los procesos de administración y desarrollo del talento humano.

\section{Agradecimientos}

A la Gerente, la jefe de talento humano, personal administrativo y asistencial de la clínica Corposalud S.A.S de la ciudad de Pasto, por la colaboración y disposición prestada durante el desarrollo de este estudio. De igual manera, se agradece al Programa de Psicología de la Universidad de Nariño y a los estudiantes del Programa de Psicología del campo organizacional durante el periodo comprendido entre el semestre B de 2015 al semestre B de 2016 por el apoyo brindado y los aportes para el desarrollo de la investigación.

\section{Conflicto de intereses}

Los autores declaran que no existen conflictos de intereses.

\section{Referencias}

1. López P, Díaz Z, Segredo AM, Pomares Y. Evaluación de la gestión del talento humano en entorno hospitalario cubano. Revista Cubana de Salud Pública. 2017;43(1): 3$15 . \quad$ Disponible en: http://scielo.sld.cu/scielo.php?script=sci_arttext\&pid= S0864-34662017000100002\&lng=es\&tlng=es.

2. Centurión, RZ. Gestión de calidad, formalización, competitividad, financiamiento, capacitación y rentabilidad en micro y pequeñas empresas de la provincia del Santa (2013). In Crescendo. 2015;6(1):146-165. Disponible en: http://revistas.uladech.edu.pe/index.php/increscendo /article/view/829/489

3. Franco G, Jaramillo D, Barreneche J. Modelo de capacitación de tecnología biomédica para clínicas y hospitales de tercer nivel, enfocado en personal asistencial. Revista ingeniería biomédica. 2015;9(18):139-144. Disponible en http://www.scielo.org.co/pdf/rinbi/v9n18/v9n18a17. pdf
4. Blanch JM. Calidad de vida laboral en hospitales y universidades mercantilizados. Papeles del Psicólogo. 2014;35(1):40-47.

5. Pérez M, Vela MJ, Abella S, Martínez Á. El Enriquecimiento Trabajo-Familia: Nuevo Enfoque en el Estudio de la Conciliación y la Satisfacción Laboral de los Empleados. Universia Business Review. 2015;(45):1633. Disponible en: http://www.redalyc.org/articulo.oa?id=43335414001

6. Arias WL, Arias G. Relación Entre el Clima Organizacional y la Satisfacción Laboral en una Pequeña Empresa del Sector Privado. Ciencia \& trabajo. 2014;16(51):185-191. Disponible en: https://dx.doi.org/10.4067/S071824492014000300010

7. Yepes M, Romeo M, Berguer R. Relación del personal sanitario con su organización y su trabajo: Un estudio cross-cultural. Rev Saúde Pública. 2016;50(18):1-4.

8. Domínguez LR, Ramírez AF, García A. El clima laboral como un elemento del compromiso organizacional. Revista Nacional de administración. 2013;4(1):59-70.

9. González L, Guevara E, Morales G, Segura P, Luengo C. Relación de la satisfacción laboral con estilos de liderazgo en enfermeros de hospitales públicos, Santiago, Chile. Ciencia y enfermería. 2013;19(1):11-21. https://dx.doi.org/10.4067/S071795532013000100002

10. Pedraza L, Socarras X, Jiménez M, Romero I. Estudio del clima organizacional en una empresa prestadora de servicios en salud ocupacional de la ciudad de Santa Marta, Colombia. Revista Clío América. 2014;8(15):4774. Disponible en: http://oaji.net/articles/2016/31671472231919.pdf

11. Báez J. La participación de los trabajadores en la empresa, el caso de Irlanda. Revista de economía pública, social y cooperativa. 2011; 1(70): 127-148.

12. Jiménez $\mathrm{S}$. Factores psicosociales y salud: de vida laboral a factor de riesgo. Rev Esp Comun Salud. 2014;5(2):134148. Disponible en: https://erevistas.uc3m.es/index.php/RECS/article/view/3340/ 1992

13. Guerrero J, Sánchez J. Factores protectores, estilos de vida saludable y riesgo cardiovascular. Psicología y Salud. 2015;25(1):57-71. Disponible en: http://revistas.uv.mx/index.php/psicysalud/article/vi ew/1339/2462

14. Díaz D, Hernández E, Isla R, Delgado N, Díaz L, Rosales C. Factores relevantes para aumentar la precisión, la viabilidad y el éxito de los sistemas de evaluación del desempeño laboral. Papeles del Psicólogo. 2014;35(2):115-121. Disponible en: http://www.redalyc.org/html/778/77831095004/

15. Sánchez H, Calderón V. Diseño del proceso de evaluación del desempeño del personal y las principales tendencias que afectan su auditoría. Pensam. Gest.2012;32(1):5482.

16. Araneda M, Pedraja L, Rodríguez E. Innovación y empresas: un análisis de sus características a nivel regional. Idesia. 2016;34(1):69-75. Disponible en: <http://www.scielo.cl/scielo.php?script=sci_arttext\&pi $\mathrm{d}=$ S0718-34292016000100008\&lng=es\&nrm=iso>. 
ISSN 0718-3429. http://dx.doi.org/10.4067/S071834292016000100008.

17. Castañeda M, Hernández L, Ramos J. Una práctica innovadora en la capacitación empresarial. Rev. Cubana Edu. Superior. 2016;35(3):4-14. Disponible en: <http://scielo.sld.cu/scielo.php?script=sci_arttext\&pid $=$ S0257-43142016000300001\&lng=es\&nrm=iso $>$. ISSN 0257-4314

18. Sapién A, Piñón A, Gutiérrez L. Capacitación en la empresa mexicana: un estudio de formación en el trabajo Civilizar. Ciencias Sociales y Humanas. 2014;14(27):123-134. Disponible en http://www.redalyc.org/articulo.oa?id=10023571600 7

19. Quintana MO, Sáez K, Figueroa C, García JA, Salazar RE, Tinajero RM, Padilla M, Valle C. Calidad de vida laboral de enfermeras de un hospital privado. Revista de ciencias biológicas y de la salud. 2016;18(2):34-38. 